\title{
The need to change the organization of athletes psychological training for competitions
}

\author{
Alexey Nikolaev*, Natalia Shlat, Irina Kolbasova, and Julia Timofeeva \\ Pskov State University, 180000, Pskov, Russia
}

\begin{abstract}
The article considers the arguments about the need to rethink the concept of athletes' psychological training for the competition. The idea of the article is the shift to the teaching the means of individual psychological training of athletes for the competition. The accuracy of this concept is grounded on the empirical data. The article presents the data of the comparative analysis of employing by athletes the means of psychological training for the competition and opinion of coaches about it. The authors explain the reasons why coaches do not prepare football players psychologically in the process of physical, technical, and tactical training. 5 reasons to explain it are considered in the article. In the process of coaches' training they are taught to regulate the psychological condition and behavior of athletes, but they are not taught how to train the athletes to do that themselves. The necessity of training football players in the means of self-regulation of their psychological condition for the matches has been proved.
\end{abstract}

\section{Introduction}

Psychological training of athletes both for a sports career in general and for a particular competition should be carried out by a psychologist of a sports team. However, this is true for theory. In practice, currently, one can hardly meet a psychologist even in highperformance sports. In this case, the question arises: who should psychologically prepare athletes for a competition if there is no psychologist? The answer would seem obvious - the coach. If the coach provides physical, technical and tactical training, then the psychological training of athletes should not be an exception. However, we can confidently say that coaches practically do not carry it out in their work [1-3]. In this case, another question arises: why? If the coach has prepared the athletes physically, technically and tactically, the athlete can only use his sports capacity. It is obtained in the training process. So why is psychological training an exception? Why don't athletes have the same psychological capacity?

There are several answers to this question.

Firstly, if other types of athletes training for the competition will be required, then psychological training may not necessarily be required; it will be needed if the psyche of the athlete gives any kind of «failure».

\footnotetext{
Corresponding author: nialo@ mail.ru
} 
Secondly, physical, technical and tactical training are distinguished by a certain stability. And if, for example, «physics», as the coaches say, is up to certain standard, then the athlete will reach his result; however, the human psyche is sensitive and dynamic. So the sporting result can suddenly «float away».

Thirdly, indicators of other types of preparedness are usually indisputable, they can be easily measured. It cannot be said with confidence about the indicators of psychological preparedness, that is, its level is hidden from the coach (and only a psychologist can determine it by means of special and comprehensive psychodiagnostics).

Fourth, the need for other types of athletes' preparedness has become an axiom. It has not happened to psychological training; the latter is traditionally carried out by a psychologist, and in his absence - by a coach.

Fifth, which follows from the fourth: the coaches would be happy to carry out the psychological training of their athletes, but they do not know how - nobody taught them to do this; during their sports activities no one taught them either. Both coaches and athletes were not taught the methods of independent psychological training.

In a successful performance, an athlete shows, for example, an adequate technique of performing movements in his sport because he was taught by the coach, he shows what he is being trained in. He shows what he can, and in the case of a psychological difficulty, he cannot use any psychological device for himself - he is not being trained in this. How, then, in such a situation does an athlete eliminate psychological difficulties, or mobilize himself? There are two answers to this question: he has summarized the experience of the psychological attitude and may have formed a behavior style in difficult but typical situations; a coach or, unlikely, a psychologist helped.

In any case, he «lives by his wits» or with some help, but does not use any of what he has been previously taught, unlike physical, technical and tactical training. In such circumstances, it would be advisable for the coaches not to invite a psychologist in case of emergency psychological assistance, but to invite athletes for special, individually implemented psychological assistance training.

\section{Materials and methods}

If the coach has to perform the psychological training of athletes for a particular performance, then it can be assumed that he has a set of tools for such training of athletes. However, again, we can assume that this set of tools was obtained from personal experience and, probably, from the experience of the practical activities of his colleagues. This is true, since even in training and continuing education programs for coaches, the issue of training athletes in self-regulation tools is most often indicated by one paragraph: «Improving the regulatory mechanisms of the psyche of athletes». This is one of 6 tasks of general psychological training $[1,4,5]$.

In the process of future coaches training, the solution to this problem is not about training athletes to use the means of regulating their own condition and behavior. It is about the means the coach can use in the process of special psychological training of the athlete for the competition [5]. Coaches are taught to regulate the mental state and behavior of athletes, but are not taught how to train the athletes to do that themselves. For example, such a tool as autogenic training is very effective. However, it is hardly ever used in sports. Why? Because, firstly, it's the same as auto-training. It involves conducting training by the athlete himself. Secondly, it's necessary to train the athletes to perform the auto-training sessions. The coach is unlikely to be able to do them.

Introductory work with training programs for athletes shows that such types of training as physical, technical and tactical are carried out constantly and in accordance with the curriculum. However, in spite of the fact that the coaches most often see the failure in the 
athletes' performances for psychological reasons (they say, the athlete was to blame himself, «he could not get together»), there is no place for psychological training similar to its other types. Even in plans and in training programs for athletes, as a rule, it is not indicated.

Of course, an athlete can get psychological help from a coach, but he has not been taught how to independently solve the psychological difficulties. The psychological training of athletes aimed at mastering the means of self-improvement can be quite wide. It may include the use of all available today means of sports activities psychological support.

Do athletes feel the need for individual psychological training? Previously, such a study was conducted and it showed that the indicators of «the needs of athletes in training for self-regulation of the mental pre-competitive condition are above ... the average level», and that «the opinion of coaches regarding the needs of athletes in self-improvement of the regulatory mechanisms of the psyche is erroneous; coaches tend to significantly underestimate these needs» $[6, \mathrm{p} .131]$.

If this is the case, then the need for training coaches in training tools for the psychological training of athletes is due to two reasons: the availability of such a need for athletes and the lack of means for its implementation [7-9].

The goal of this empirical study is to identify differences between the means used by athletes in the implementation of psychological self-training for competitions, and coaches' assessments of the activity of using these means. In other words, the goal of the study is to determine the coaches' awareness about the activity of athletes in their psychological selftraining for competitions.

The selection of examinees included 36 football players of youth age from sports teams in the city of Pskov, Russia and the region (4 teams) and their 6 coaches. The written survey method was used - a scaled version of the questionnaire with 9 points detection. The stimulus material was the actions of the players to use those means that can be used for selfprevention (means No. 1 and 2) and self-regulation of the pre-match condition. Athletes and coaches evaluated 12 actions of 36 athletes (these actions are reflected in the chart below):

1) using the exercise method (creating repeatability of competitive situations to adapt to them);

2) simulation of competition conditions;

3) turning off and switching attention;

4) performing slow or intense physical work;

5) rational therapy;

6) the method of emotional discharge;

7) a change in motivation for performance;

8) relaxation technique;

9) breathing exercises;

10) autogenic training;

11) sleep induction and / or rest;

12) the acquisition and use of an individual style of emotional self-regulation (prematch settings).

\section{Results and discussion}

The following empirical results of athletes actions degree indicators aimed at selfprevention (means No. 1 and 2) or self-regulation of the pre-match mental condition were obtained. In addition, estimates are given here by the coaches of the athletes' actions regarding the use of the following self-prevention means (means No. 1 and 2) or selfregulation means.

The validity of differences was determined with the $\chi^{2}$ criterion (chi-square conjugation 
coefficient) for four-field charts: the number of players with a high frequency of using each of the self-regulation means and with a low frequency of that; the number of players with a high frequency of using each of the means, according to the coach, and with a low frequency (see the Table 1).

Table 1. The use of self-prevention means and self-regulation by athletes of the pre-match condition and behavior, as well as their assessment by coaches

\begin{tabular}{|l||c|c||c|c||c||c|c|}
\hline \multirow{2}{*}{ Means of self-regulation: } & \multicolumn{2}{|c||}{ Athletes } & \multicolumn{2}{c||}{$\begin{array}{c}\text { Their } \\
\text { coaches }\end{array}$} & \multirow{2}{*}{$d$} & \multirow{2}{*}{$\chi^{2}$} & \multirow{2}{*}{$p$} \\
\cline { 2 - 6 } & $\bar{x}_{\mathbf{c}}$ & $\begin{array}{c}v_{\mathbf{c}} \\
\%\end{array}$ & $\bar{x}_{\mathrm{T}}$ & $\begin{array}{c}v_{\mathbf{T}} \\
\%\end{array}$ & & & \\
\hline \hline 1) method of exercise & 3,32 & 9,9 & 2,83 & 43,8 & 1,51 & 3,98 & 0,05 \\
\hline 2) modeling of conditions & 0,65 & 11,2 & 0,33 & 27,9 & 0,32 & 1,06 & - \\
\hline 3) switching of attention & 4,84 & 23,1 & 2,12 & 38,3 & 2,72 & 12,2 & 0,001 \\
\hline $\begin{array}{l}\text { 4) alteration } \\
\text { of speed of actions }\end{array}$ & 1,18 & 10,4 & 0,98 & 14,4 & 0,20 & 0,84 & - \\
\hline 5) rational therapy & 5,13 & 16,1 & 2,08 & 32,0 & 3,05 & 21,7 & 0,001 \\
\hline 6) emotional release & 3,66 & 18,8 & 2,15 & 27,3 & 1,51 & 4,21 & 0,05 \\
\hline 7) change of motivation & 1,25 & 23,2 & 2,87 & 25,4 & $-1,62$ & 4,75 & $-0,05$ \\
\hline 8) relaxation technique & 5,02 & 9,8 & 2,15 & 28,5 & 2,87 & 5,12 & 0,05 \\
\hline 9) breathing exercises & 2,65 & 25,5 & 1,48 & 31,4 & 1,23 & 3,88 & 0,05 \\
\hline 10) autogenic training & 0,14 & 47,9 & 0,44 & 32,7 & $-0,30$ & 0,35 & - \\
\hline 11) sleep induction & 0,55 & 32,8 & 0,22 & 33,7 & 0,33 & 0,41 & - \\
\hline 12) individual style & 5,26 & 16,7 & 2,15 & 38,7 & 3,11 & 22,4 & 0,001 \\
\hline \hline \multicolumn{1}{|c||}{ Average values: } & 2,80 & 20,4 & 1,65 & 31,2 & - & - & 0,02 \\
\hline \hline
\end{tabular}

The data obtained say a lot. For clarity, we present a diagram on the use of special psychological self-training tools for athletes and the opinions of coaches on this (see the figure 1 below).

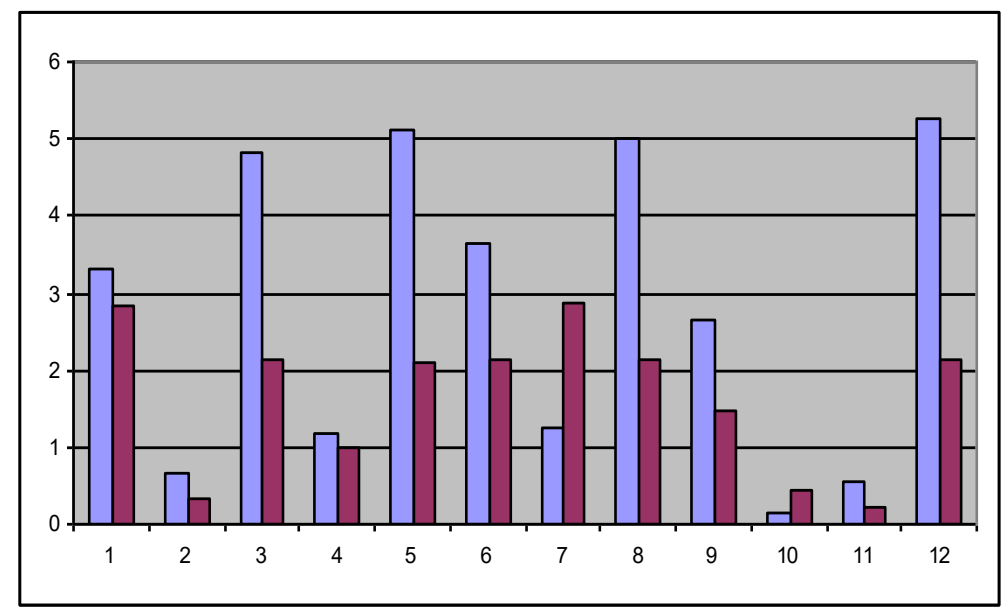

Fig. 1. The use of psychological self-training means by sportsmen and the opinions of their coaches.

Explanations: 1-12 - Means of self-regulation; psychological self-training means are placed under the same numbers as in the chart; row 1 - the actual use of means, row 2 - their use, according to coaches' opinion; the use of self-regulation tools was evaluated on a scale with 9 points detection (in the figure, the degree is limited to 6 points). 
1. In general, athletes make little use of the psychological self-training means for competitions (means of self-prevention and self-regulation of behavior and condition), only 2.8 points out of 9 , that is, less than a third. However, the use of various means is very diverse - from 0.14 points (using autogenic training) to 5.26 points (individual style of prestart attitude); the coefficient of variation of the means used is $67.9 \%$.

We can differentiate most often used means of players special psychological selftraining before the match:

- individual style of self-training;

- rational therapy;

- relaxation technique;

- and switching attention.

The following means are used rarely:

- autogenic training;

- sleep induction;

- modeling the conditions of the upcoming match;

- performing slow or intense physical work as a means of self-regulation;

- and a change (often decrease) in the level of motivation to win.

Moderate coefficients of variation are obtained for most means of self-regulation. However, the coefficient of the means variation is very high $(52.7 \%)$, this suggests that some of the means are used by most athletes, but the other part is used by the minority.

2. Coaches rate the activity (frequency) of using the means of special psychological self-training of football players for matches very low - only 1.65 points out of 9 .

Most often, athletes, according to the coaches' opinion, use the following means of their psychological self-regulation: change in motivation (apparently, therefore, coach's express dissatisfaction with unsuccessful players); exercise the method to accelerate adaptation to conditions; relaxation method; and the use of an individual style of psychological selfregulation. (However, not a single tool scored higher than 3 points out of 9 ). The coaches ranked the lowest use of the following means: modeling the conditions for matches; rest; and autogenic training.

3. Comparison of the coaches' opinions on the use of self-regulation and their actual use by athletes revealed significant differences - on average, according to coaches' opinion, athletes are much less likely to use means of independent psychological training.

The greatest differences were found in the following indicators (where coaches underestimated the actions of athletes):

- the use of an individual style of psychological self-training;

- rational therapy method;

- relaxation method.

The use of the method of changing the strength of motives for successful performance is rated by coaches above its real application by players.

The greatest differences (at $99.9 \%$ confidence) were found by the following three indicators (by decreasing): the use of an individual style of emotions (pre-match psychological settings) self-regulation; rational therapy; and such a means of self-regulation as disconnecting and switching attention from the upcoming match.

\section{Conclusion}

In general, it can be noted that athletes use little means of independent psychological training for matches, and coaches evaluate their preparation even lower. These two circumstances indicate that athletes need to be specially trained in the means of an unfavorable mental condition self-diagnosis and in the means of their self-regulation.

The possible difficulties noted above when changing the form of psychological training 
organization do not withstand the scrutiny for the following reasons.

1. The opinion that the psychological preparedness of athletes may not be in demand is a mistake. In the process of special psychological training, one can only come closer to full preparedness. But it is impossible to achieve $100 \%$ preparedness.

2. It is also erroneous that if the human psyche is sensitive, then the athletes' ability to conduct self-regulation may not be required. On the contrary, in changing external conditions and situations, urgent and quick self-regulation of the mental state may be required.

3. If indicators of psychological readiness are not obvious, then this does not mean that it is unnecessary to diagnose them. On the contrary, it is advisable to use the full capacity of express diagnostics.

4. The traditional implementation of psychological training of athletes by a coach, and sometimes by a psychologist, does not exclude their psychological self-training. This is a way of increasing the resource in the process of achieving the psychological preparedness of athletes.

5. If coaches are not able to teach athletes how to conduct psychological training, then acting coaches need to be retrained, and future coaches need to be taught.

The revision of the psychological service organization in sports should be the main means of psychological preparation of athletes for the competition.

Coaches should be prepared to provide psychological assistance to athletes, but primarily, they should be prepared for training athletes in psychological self-training for sports performances.

\section{References}

1. G.D. Gorbunov, The psychopedagogical of the sport (Soviet sport, Moskva, 2007)

2. G.D. Gorbunov, Theory and practice of physical culture, 12, 15-17 (1996)

3. G.D. Babushkin, Specifics of the trainer's activity (OGIFK, Omsk, 1985)

4. G.D. Babushkin, Psychological support of sports activity: monograph (Sibgufk publishing house, Omsk, 2006)

5. G.V. Soldatova, Theory and practice of educational process management: pedagogical, social and psychological issues: Bulletin of BPA, 1444, 54-57 (2013)

6. A.N. Nikolayev, Physical Education and sports. Olympic education, 2, 129-131 (2019)

7. G.I. Savenkov, Psychological preparation of an athlete in the modern system of sports training: study guide (Physical culture, Moskva, 2006)

8. A.S. Kuznetsov, Z.M. Kuznetsova, Russian Journal of Physical Education and Sport, 14(4), 5-7 (2019)

9. V.G. Savitsky, Theory and practice of physical culture, 6, 40-43 (2006) 